\title{
AN UNCOUPLED OSCILLATOR MODEL FOR EVOKED POTENTIAL DYNAMICAL MODELLING
}

\author{
Richard B. Reilly, Member IEEE, Tomás E. Ward and Annraoi M. dePaor \\ e-mail : rreilly@elecmag3.ucd.ie
}

Dept. of Electronic and Electrical Engineering, University College, Dublin, Ireland.

\begin{abstract}
A mathematical model for evoked potentials is outlined. The model has been developed by considering phase synchronisation of the underiying neurological processes. The model, consisting of an ensemble of uncoupled linear oscillators with a gaussian distribution of frequencies is shown to reproduce the typical response of $\mathrm{P100}$ visual evoked response. The model structure is parallel in form and is considered to be physiologically realistic. It is also shown that the calculated behaviour of the ensemble can be generated by $2^{\text {nd }}$ order linear differential equation with time varying coefficients, thus highlighting the fact that entirely different physical structures can generate identical responses.
\end{abstract}

\section{INTRODUCTION}

The analysis of EEG data for the presence of evoked potentials has traditionally been carried out by a signal averaging process. However, a number of methods have been proposed which employ the information contained within the pre-stimulus interval to aid the identification process [1] [2]; it is generally assumed that the evoked response is an additive contribution to the on-going spontaneous EEG activity.

However, is has been suggested that the external stimulus acts to phase-modify the spectral components of the spontaneous activity [3]. It has been further suggested that evoked potentials and event related potentials reflect external stimulus and endogenous synchronisation, frequency stabilisation, frequency selective enhancement and phase re-ordering of the on-going EEG [4]. It has also been reported that 750 milliseconds following auditory stimulus any phase-control of spontaneous EEG activity diminishes, allowing fully spontaneous activity to be restored [5]. As a point of interest one of the authors was involved in the development of a model of the autoregulation in the kidney based on uncoupled but nonlinear oscillators [6].

The approach taken involves analysis of evoked responses in the frequency domain. An investigation of Fourier harmonic components show that the phase angle of a given component becomes a random variable, due to the noise content of the measured waveform. This phase angle has a uniform distribution from $0^{\circ}-360^{\circ}$ if the component is composed of solely of noise. However, it has been reported that the presence of an evoked response will cause an aggregation of phase values in some angular region when repeated measurements are performed, with resulting non-uniformity of the phase angle distribution [7]. Such non-uniformity has been employed as a detection criterion for the presence of auditory brainstem evoked potentials, resulting in a reduction of stimulation administered to the subject.

\section{MODEL STRUCTURE}

A model composed of linear oscillators has been developed to simulate the synchronisation of phases. Considering $\sin (\omega t)$, where $\omega$ is a random variable with probability density function

$$
p(\omega)=\frac{1}{\sigma \sqrt{2 \pi}} e^{-\frac{(\omega-\mu)^{2}}{2 \sigma^{2}}}
$$

The expected value of $\sin (\omega t)$ is

$$
\begin{gathered}
y(t)=\int_{\omega=-\infty}^{\infty} \sin (\omega t) \cdot \frac{1}{\sigma \sqrt{2 \pi}} e^{-\frac{(\omega-\mu)^{2}}{2 \sigma^{2}}} \cdot d \omega \\
y(t)=\frac{1}{2 j \sigma \sqrt{2 \pi}} \int_{\omega=-\infty}^{\infty}\left\{e^{j \omega x}-e^{-j \omega t}\right\} e^{-\frac{(\omega-\mu)^{2}}{2 \sigma^{2}}} \cdot d \omega
\end{gathered}
$$

Since $p(\omega)$ is a probability density function,

$$
\int_{-\infty}^{\infty} p(\omega) d \omega=1
$$

independent of $\mu$ and $\sigma$. If the first integrand is denoted as eqn. (5)

$$
I_{1}=\frac{1}{\sigma \sqrt{2 \pi}} \int_{\omega=-\infty}^{\infty} e^{j \omega \alpha} e^{-\frac{(\omega-\mu)^{2}}{2 \sigma^{2}}} \cdot d \omega
$$

the second integrand is simply the same with $t$ replaced by $-t$. Consider the integrand

$$
e^{\frac{-(\omega-\mu)^{2}+j 2 \sigma^{2} \alpha x}{2 \sigma^{2}}}
$$

This can be configured into the form of eqn. (7). 


$$
\begin{gathered}
e^{j \mu t} \cdot e^{-\frac{\sigma^{2} t^{2}}{2}} \cdot e^{\frac{-\left(\omega-\mu-j \sigma^{2} t\right)^{2}}{2 \sigma^{2}}} \\
\therefore I_{1}=e^{j \mu t} \cdot e^{-\frac{\sigma^{2} t^{2}}{2}} \frac{1}{\sigma \sqrt{2 \pi}} \int_{-\infty}^{\infty} e^{\frac{-\left(\omega-\mu-j \sigma^{2} t\right)^{2}}{2 \sigma^{2}}} \cdot d \omega
\end{gathered}
$$

which from eqn. (7) evaluates to

$$
\begin{gathered}
I_{1}=e^{j \mu t} \cdot e^{-\frac{\sigma^{2} t^{2}}{2}} \\
\therefore \quad y(t)=e^{\frac{-\sigma^{2} t^{2}}{2}} \cdot \sin \mu t
\end{gathered}
$$

This is the result for resetting all the phases of the oscillators to zero. For $n$ oscillators the expected sum will be

$$
y(t)=n \cdot e^{\frac{-\sigma^{2} t^{2}}{2}} \cdot \sin \mu t
$$

This function is graphed in Fig. 1 for $\mu=10$ and a range of $\sigma$ values.

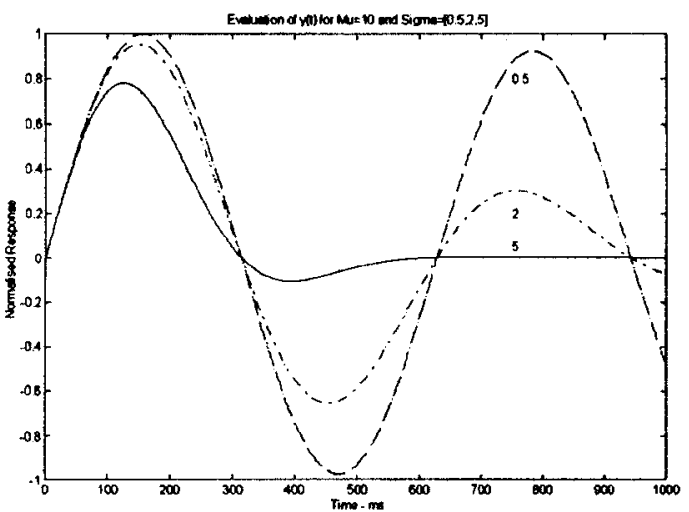

Fig. 1. Graph of eqn. (11) for $n=1, \mu=10$ and a range of $\sigma$ values.

A differential equation solved by this $y(t)$ is

$$
\frac{d^{2} y}{d t^{2}}+2 \sigma^{2} t \frac{d y}{d t}+\left[\sigma^{2}+\mu^{2}+\sigma^{4} t^{2}\right] y=n \mu \alpha(t)
$$

This differential equation describes a linear time-variant system. One solution is for $y(0)=0 ; \frac{d y}{d t}(0)=0$.

\section{EXPERIMENTATION AND ANALYSIS}

Investigation of the full field monocular pattern shift reversal Visual Evoked Potential (VEP) was carried out [8]. A sample rate of $2000 \mathrm{~Hz}$ was employed, with the checkerboard pattern being reversed at a rate of $1 \mathrm{~Hz}$. The P100 waveform was detected following ensemble averaging of 100 trails. Using a 2-D assembly of oscillators $(15 \times 15)$ a response similar to the experimentally measured P100 was observed from the summation of the outputs on resetting the phases, Fig.2. This simulated response can be described analytically by eqn. (11). Thus, a measure of the variance of the oscillator frequencies can be obtained. The P100 has been modelled by assuming that our measurement system observes the outputs of two communities of oscillators, in the vicinity of active and reference electrodes, reset in sequence by a wave travelling through the brain. Thus

$$
P 100=y(t)
$$

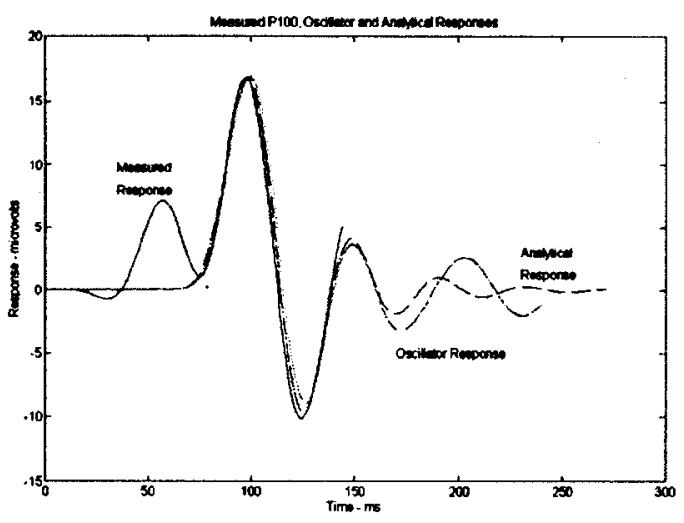

Fig. 2. Measured P100, Oscillator and Analytical Responses

\section{Conclusion}

A model based on a parallel configuration of oscillators has been shown to produce a response similar to that of the Pl00 obtained from the full field monocular pattern shift reversal Visual Evoked Potential. A measure of the variance of the underlying neurological oscillator model can be calculated, which may then be used as a benchmark for neurological studies.

\section{REFERENCES}

1 M. Furst, A. Blau, "Optimal A Posterioir Time Domain Fitter for Average Evoked Potentials", IEEE Trans. BME, Vol-38, No.9, pp 827 833, 1991.

2 S. Nishida, M. Nakamura, H. Shibasahi, "Method of Predicting an EEG Waveform as an Aid to the Accurate Recording of Evoked Potentials", IEEE Trans. BME, Vol-38, No.9, pp 827 - 833, 1991.

3 B. McA. Sayers, H.A. Beagley, W.R. Henshall, "The Mechanism of Auditory Evoked EEG Responses", Nature, Vol. 247, pp 481-483, 1974.

4 G. Stampfer, E. Basar, "Does Frequency Analysis lead to Better Understanding of Human Event Related Potentials", Int. Journal. Neuroscience, Vol. 26, pp 181-196, 1985.

5 B. McA. Sayers, H.A. Besgley, "Objective Evaluation of Auditory EEG Responses”, Nature, Vol. 251, pp 608-609, 1974.

6 A.M. dePaor, P. Timmons, "A Feedback Oscillator Model for Cinculatory Autoregulation”, Int. J. Control, Vol. 43, No. 2, pp $679-688,1986$.

7 N.I. Bachen, "Detection of Stimulus Related (Evoked Response) Activity in Electroencephalogram (EEG)", IEEE Trans. BME, Vol33, No.6, pp 506 - 571, 1986.

8 A.M.Halliday (ed.), Evoked Potentials in Clinical Testing, Clinical Neurology and Neurosurgery Monographs, Churchill Livingstone, Edinburgh, 1982. 